\title{
Methodology of Autolanding Trajectory Design for Reusable Launch Vehicle
}

\author{
Shuai Huang ${ }^{1, a}$, Ran Zhang ${ }^{2, b}$, Hong Cai ${ }^{1, c}$ and Wenming Nie ${ }^{2, d}$ \\ ${ }^{1}$ College of Aerospace Science and Engineering, National University of Defense Technology, \\ Changsha 410073, China; \\ ${ }^{2}$ School of Astronautics, Beihang University, Beijing 100191, China. \\ aase_huang@hotmail.com, bzhangran@buaa.edu.cn, chcai@nudt.edu.cn, dwenming0912@buaa.e \\ du.cn
}

Keywords: RLV, autolanding, trajectory design.

\begin{abstract}
A trajectory design methodology is developed for autolanding phase of RLV (Reusable Launch Vehicle) based on iterative correction algorithm in this paper. According to the inherent traits in phase of unpowered landing of RLV, geometric structure of trajectory and dynamic characteristics of the aircraft are studied in detail. And mathematical descriptions of the trajectory as well as the selection principle and calculation formula of feature parameters are given. Compared with the traditional design methods of trajectory, this methodology is simple and rapid. The simulation results also demonstrate the feasibility, effectiveness, and superiority of the algorithm.
\end{abstract}

\section{Introduction}

Autolanding is the last phase in the whole flight process of RLV, and the design and optimization of trajectory in this stage is the basic premise to ensure the flight mission ending in a satisfactory way ${ }^{[1,2,3]}$. Traditional design of trajectory, mainly aiming at powered landing, does not consider the dynamic characteristics of aircrafts too much, for the lift drag ratio of the vehicle can be changed by adjusting the engine throttle to adapt to different uncertainties and external disturbances. However, for RLV or other unpowered vehicles in the approach and landing phase, their ability to adapt the uncertainty of energy state is not enough ${ }^{[3]}$. So in other to ensure the security landing as the expected index, the geometric constraints of the trajectory must be combined with the dynamic characteristics of the aircraft and related vehicle state parameters along the trajectory should be calculated through the simulation to determine whether the landing program meets the requirements when designing the autolanding trajectory of aircrafts.

At present, unpowered autolanding can be achieved only on the US shuttles and hypersonic vehicles such as X-34 and X-37 in engineering fields, and the trajectory mainly consists of "steep glide slope+ circular flare+ exp. Decay+ shallow glide slope" ${ }^{\text {"4,5] }}$. It has been proved that this programme is suitable for such low lift-over-drag vehicles like RLV. On this basis, a trajectory design methodology is developed for autolanding phase of RLV based on iterative correction algorithm in this paper. According to the initial state at the automatic landing interface (ALI), the trajectory is propagated numerically using constraints of geometry and dynamics until meeting the landing requirements.

\section{Trajectory Design Methodology}

Autolanding trajectory of RLV generally begins at the automatic landing interface (ALI) whose height is about $3000 \mathrm{~m}$, and is divided into four stages: steep glide slope, circular flare, Exp. decay and shallow glide slope, as shown in figure $1^{[6,7]}$. Each segment of the trajectory with such structure has obvious physical meaning and is closely related with the dynamic characteristics of the vehicle.

In stage of steep glide slope, RLV keeps constant flight path angle and flies in state of quasi-equilibrium. The main function of this stage is to reduce and eliminate the influence of various deviations during the flight. The flight path angle is relatively large due to the small lift-to-drag of 
RLV. Circular flare is the transition stage between steep glide slope and shallow glide slope to ensure the continuity of acceleration. And the Exp. decay is introduced to realize a smooth transition of overload. Until landing on the ground, RLV is in stage of shallow glide slope with a small vertical velocity to minimize the landing impact.

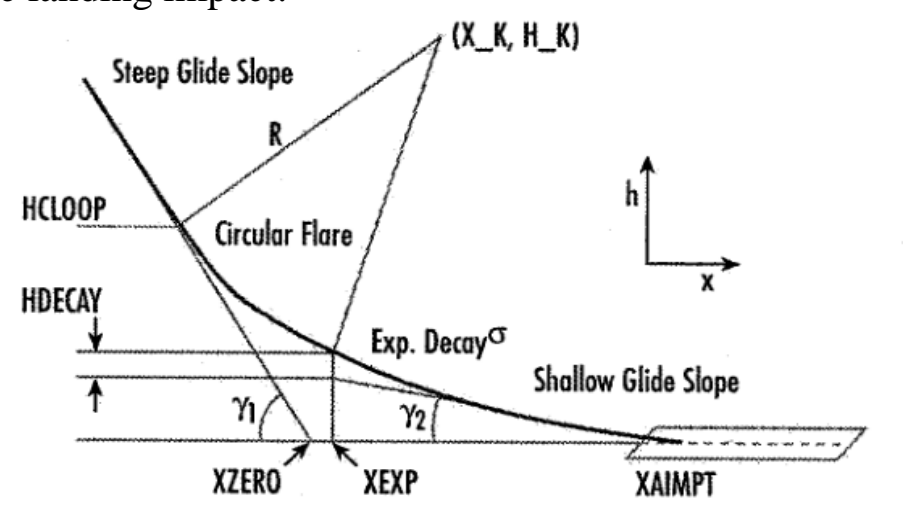

Fig. 1 Structure of auto-landing trajectory

Parameters Design of Trajectory. As shown in figure 1, the physical meanings of parameters about the trajectory in autolanding of RLV are listed in the following table:

Table 1 Instruction of trajectory feature parameters

\begin{tabular}{cc}
\hline Trajectory parameters & Physical meaning \\
\hline$\gamma_{1}$ & Flight path angle in steep glide slope \\
$\gamma_{2}$ & Flight path angle in shallow glide slope \\
$X_{A}$ & The landing target point \\
$X_{Z}$ & The intersection of extension line of steep glide slope trajectory and the ground \\
$X_{E}$ & Starting point of Exp. decay stage \\
$R$ & Radius of curvature in stage of circular flare \\
$\left(X_{K}, H_{K}\right)$ & Center coordinates of circular flare stage \\
$H_{C}$ & Initial height of circular flare stage \\
$H_{D}$ & Proportional coefficient of the exponential function \\
$\sigma$ & Convergence coefficient of the exponential function
\end{tabular}

According to the order of determination, feature parameters can be divided into two categories. The first one includes $\gamma_{1}, \gamma_{2}, x_{A}, R, h_{D}$ and $x_{Z}$, which need to be determined in advance. The rest belong to the second kind, which are determined through the first kind parameters and other constraints.

(1) Selection of the first kind parameters

$\gamma_{1}$ can be determined according to the situation that the dynamic pressure and flight path angle of RLV are constant during the stage of steep glide slope. Its solvation equations are written as:

$$
\left\{\begin{array}{l}
\frac{d q}{d h}=\left(\frac{1}{\rho} \frac{d \rho}{d h}-\frac{\rho S C_{D}}{m \sin \gamma}\right) q-\rho g=0 \\
\frac{d \gamma}{d h}=\frac{\rho}{2 \sin \gamma}\left(\frac{S C_{L}}{m}-\frac{g \cos \gamma}{q}\right)=0
\end{array}\right.
$$

Design of $\gamma_{2}$ should consider the requirement of sink rate to avoid that RLV drops too fast or the overload beyond the limitation in the final stage of landing.

$x_{A}$ can be determined randomly according to the length of runway.

The selection of $h_{D}$ is mainly based on experience and many tests will be conducted to ensure a proper sink rate in stage of Exp. decay.

$R$ should be determined according to the overload $n_{z}$ in stage of circular flare. The formula is shown as follows: 


$$
R=v_{C}^{2} /\left(g \cdot n_{Z}\right)
$$

where $v_{C}$ is the velocity of RLV at the point $h_{C}$.

The value of $x_{Z}$, corresponding to the flight distance of autolanding, determines the energy dissipation and influences the final dynamic pressure of RLV directly. Therefore, $x_{z}$ is usually adjusted and optimized continually according to the limitations of landing.

(2) Calculation of the second kind parameters

After determining the first kind parameters, other parameters can be calculated according to geometric constraints and requirements of continuity and stability.

$$
\begin{aligned}
\sigma & =\sqrt{\frac{R h_{D}}{\left[1+\left(\tan \gamma_{2}-\frac{h_{D}}{\sigma}\right)^{2}\right]^{\frac{3}{2}}}} \\
x_{K} & =\frac{\tan \gamma_{1}\left(\frac{R}{\sin \gamma_{1}}-x_{Z}\right)+x_{A} \cdot \tan \gamma_{2}-R \cos \left[\arctan \left(\tan \gamma_{2}-\frac{h_{D}}{\sigma}\right)\right]-R \cdot \tan \gamma_{2} \cdot \sin \left[\arctan \left(\tan \gamma_{2}-\frac{h_{D}}{\sigma}\right)\right]-h_{D}}{\tan \gamma_{2}-\tan \gamma_{1}} \\
h_{K} & =\tan \gamma_{1}\left(x_{K}-x_{Z}+\frac{R}{\sin \gamma_{1}}\right) \\
h_{C} & =h_{K}-R \cos \gamma_{1} \\
x_{E} & =x_{K}+R \sin \left[\arctan \left(\tan \gamma_{2}-\frac{h_{D}}{\sigma}\right)\right]
\end{aligned}
$$

Mathematic Description of Trajectory. To achieve the algorithm simulation, expression of $h=f(x)$ and relative information about $f^{\prime}(x)$ and $f^{\prime \prime}(x)$ are needed, as well as the flight path angle and its change rate, which are written as:

$$
\begin{aligned}
& \gamma=\arctan \left(\frac{d h}{d x}\right) \\
& \frac{d \gamma}{d h}=\frac{d}{d h}\left[\arctan \left(\frac{d h}{d x}\right)\right]=\frac{1}{1+\left(\frac{d h}{d x}\right)^{2}} \frac{d^{2} h}{d x^{2}} \frac{1}{\frac{d h}{d x}}
\end{aligned}
$$

(1) Steep glide slope

$h=\left(x-x_{Z}\right) \tan \gamma_{1}$

(2) Circular flare

$h=h_{K}-\sqrt{R^{2}-\left(x-x_{K}\right)^{2}}$

(3) From Exp. decay to shallow glide slope

$h=\left(x-x_{A}\right) \tan \gamma_{2}+h_{D} \exp \left(\frac{x_{E}-x}{\sigma}\right)$

As space is limited, the specific expressions of relevant variables are not given here.

Simulation Algorithm of Trajectory. According to the dynamic equations in longitudinal plane of RLV, we can derive that

$$
\left\{\begin{array}{c}
\frac{d q}{d h}=\left(\frac{1}{\rho} \frac{d \rho}{d h}-\frac{\rho S C_{D}}{m \sin \gamma}\right) q-\rho g \\
q=\frac{\rho m g \cos \gamma}{\rho S C_{L}-2 m \frac{d \gamma}{d h} \sin \gamma}
\end{array}\right.
$$

The previous section has shown that $\gamma(h)$ and $\gamma^{\prime}(h)$ can be uniquely determined after knowing the geometrical relationship of trajectory. And atmospheric density is calculated as follows: 


$$
\rho=1.2266 \exp \left(-\frac{h}{7315.2}\right)
$$

Thus, in the process of autolanding, atmospheric density, flight path angle and their derivatives are functions of altitude. So, above equations can be written as:

$$
\left\{\begin{array}{c}
\frac{d q}{d h}=f_{1}(q, \alpha, h) \\
q=f_{2}(\alpha, h)
\end{array}\right.
$$

Through the above-mentioned process, it is obviously that dynamic pressure is the only variable, while the angle of attack is the control variable and altitude is the independent variable. Thus, a differential-algebraic system is established and its solution method is shown as figure 2. For a given trajectory, the initial state parameters including height, pressure, and angle of attack are given as a basis for iterative calculation. Then the trajectory is divided into several discrete points to predict the angle of attack of the next point. However, the accurate calculation is based on the constraints of dynamic pressure. The angle of attack making two values of dynamic pressure equal to each other is the required control variable.

Given the angle of attack, $q_{1}$ can be calculated by integrating the differential equation. And $q_{2}$ is calculated using the algebraic expression directly. Euler method is used here.

$$
\begin{aligned}
& q_{1}(h+\Delta h)=q(h)+\frac{\Delta h}{2}\left[\frac{d q}{d h}(h)+\frac{d q}{d h}(h+\Delta h)\right] \\
& q_{2}(h+\Delta h)=f_{2}[\alpha(h+\Delta h), h+\Delta h]
\end{aligned}
$$

The condition of ending the iteration at each point is

$$
\left|q_{1}(h+\Delta h)-q_{2}(h+\Delta h)\right| \leq \varepsilon
$$

Then, the variation of control variable (angle of attack) and state variable (dynamic pressure) of RLV along the whole trajectory can be known through the iterative calculation.

In order to meet the requirement of the final dynamic pressure, it is necessary to adjust the value of $x_{z}$ to achieve the optimization of landing trajectory. Thus, it has been transformed into a two-point boundary problem with known initial dynamic pressure and terminal dynamic pressure. The specific process of calculation is shown in figure 3.

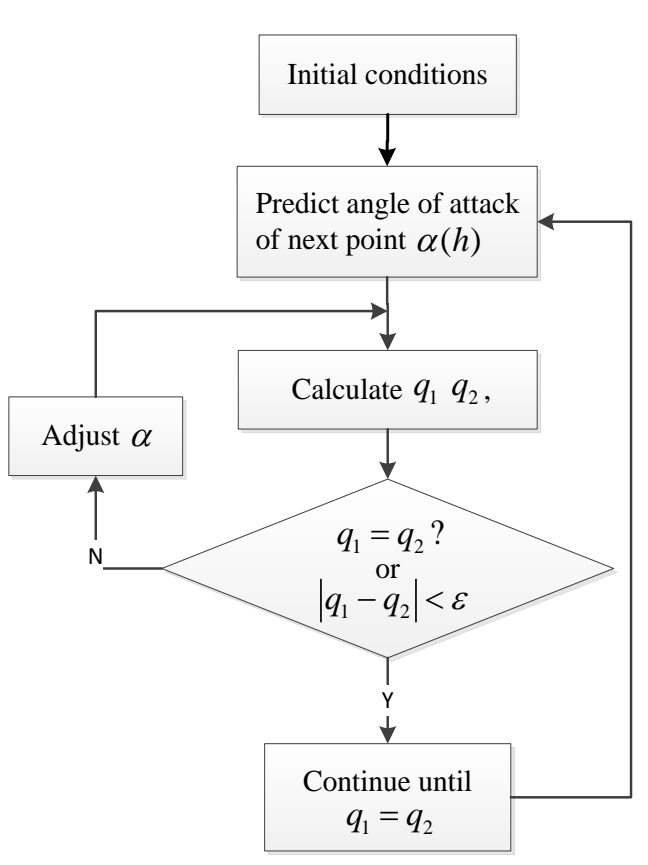

Fig. 2 Flow chart of trajectory derivation

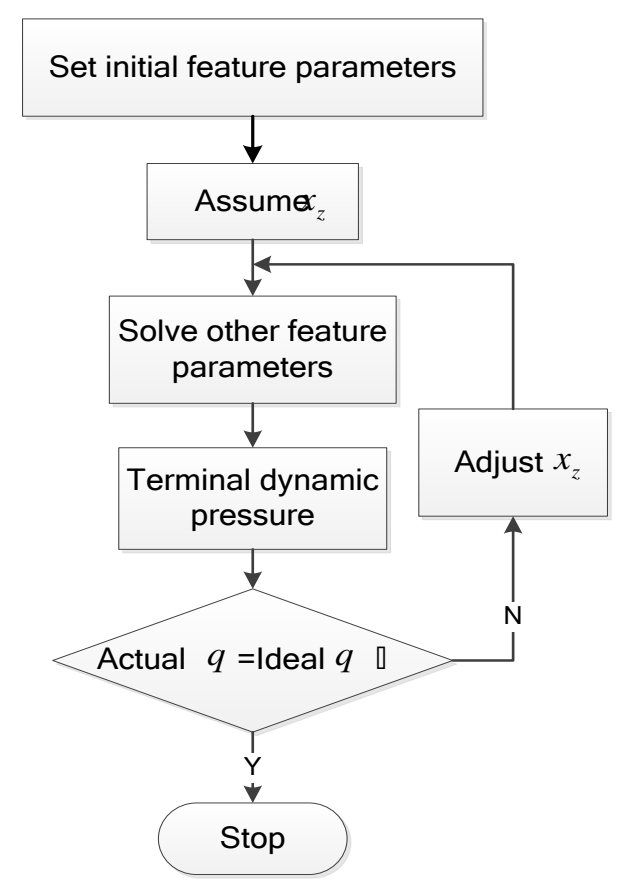

Fig. 3 Flow chart of trajectory optimization 


\section{Simulation Results}

HL20 is taken as the example in this section to design and optimize its trajectory in phase of autolanding using the methodology introduced in last section.

Relative Parameters of the Vehicle and Constraints of Trajectory Design. Relative parameters needed about HL20 are listed below:

Table 2 Relative parameters of HL20

\begin{tabular}{cc}
\hline parameter & value \\
\hline Mass $m$ & $11739 \mathrm{~kg}$ \\
Relative area $S$ & $26.612 \mathrm{~m}^{2}$ \\
\hline
\end{tabular}

According to the real situation, initial altitude of RLV in phase of autolanding is $h_{0}=3000 \mathrm{~m}$, while initial velocity is $v_{0}=204 \mathrm{~m} / \mathrm{s}$, and terminal velocity is $v_{n}=106 \mathrm{~m} / \mathrm{s}$. So initial dynamic pressure is

$$
q_{0}=\frac{1}{2} \rho(h) v_{0}^{2}=16937 \mathrm{~N} / \mathrm{m}^{2}
$$

The ideal terminal dynamic pressure is

$$
q_{n}=\frac{1}{2} \rho(h) v_{n}^{2}=6891 \mathrm{~N} / \mathrm{m}^{2}
$$

Selection and Calculation of Trajectory Parameters. To design trajectory, $\gamma_{1}, \gamma_{2}, x_{A}, h_{D}$ and $R$ should be determined at first.

According to above analysis, $\gamma_{1}$ can be determined using equations of (1), and its equivalent equations are:

$$
\left\{\begin{array}{c}
q S C_{L}=m g \cos \gamma_{1} \\
q S C_{D}=m \sin \gamma_{1}\left(\frac{1}{\rho^{2}} \frac{d \rho}{d h}-g\right)
\end{array}\right.
$$

The flight path angle and angle of attack in phase of steep glide slope can be calculated by solving this nonlinear algebraic equations. In fact, these two angles in steep glide slope are merely dependent on height. Their small change is the result of the variety of density. According to the methodology mentioned above, the flight path angle in phase of steep glide slope should keep constant. Thus, the dynamic pressure will vary with height. And different $\gamma_{1}$ will lead to different profiles of dynamic pressure. To maintain a small change of dynamic pressure of HL20 in phase of steep glide slope, $\gamma_{1}=-17^{\circ}$ is selected here.

$\gamma_{2}$ should be determined according to the terminal velocity and sink rate. The formula is given as follows:

$$
\dot{h}=v \sin \gamma_{2}
$$

Due to the maximum value of sink rate that the landing gear of HL20 can sustain, $\gamma_{2}=-1^{\circ}$ is taken here.

As the change of flight path angle is relatively large during the segment from the stage of steep glide slope to shallow glide slope, the selection of $R$ should make in a proper range to avoid over-large acceleration in phase of circular flare. Here, $n_{z}=0.37, v_{C}=180 \mathrm{~m} / \mathrm{s}$, and $R=8926 \mathrm{~m}$.

Proportional coefficient $h_{D}$ of the exponential function represents the proportion of Exp. decay in the whole flight process. To maintain a proper sink rate, $h_{D}=20 \mathrm{~m}$ is taken here.

$x_{A}=0 \mathrm{~m}$, i.e. taking the landing point of HL20 as the origin of trajectory profile.

Optimization Design of $x_{z}$. The location of $x_{z}$ determines the telescopic of trajectory along the axis and influences the state parameters, including the terminal dynamic pressure, of each point of the trajectory. Therefore, $x_{z}$ needs to be optimized to meet constraints of landing. Feature parameters and terminal states corresponding to different $x_{z}$ are given in Table 3. 
Table 3 Feature parameters and terminal states corresponding to different $x_{Z}$

\begin{tabular}{cccc}
\hline & $x_{Z}=-1800 \mathrm{~m}$ & $x_{Z}=-2000 \mathrm{~m}$ & $x_{Z}=-2200 \mathrm{~m}$ \\
\hline$\left(x_{C}, h_{C}\right)$ & $(-3143.3,410.7)$ & $(-3355.4,414.4)$ & $(-3567.5,418.1)$ \\
$\left(x_{E}, h_{E}\right)$ & $(-1111.9,39.41)$ & $(-1324,43.11)$ & $(-1536.2,46.81)$ \\
$\left(x_{K}, h_{K}\right)$ & $(-533.5,8947)$ & $(-745.6,8950.7)$ & $(-957.7,8954.4)$ \\
Dynamic pressure $\left(\mathrm{N} / \mathrm{m}^{2}\right)$ & 8061.6 & 7251.2 & 6391.8 \\
Angle of attack $\left({ }^{\circ}\right)$ & 16.13 & 17.86 & 20.44 \\
Velocity $(\mathrm{m} / \mathrm{s})$ & 114.65 & 108.73 & 102.09 \\
Sink rate $(\mathrm{m} / \mathrm{s})$ & 2.001 & 1.898 & 1.782 \\
\hline
\end{tabular}

Due to the different $x_{z}$, the trajectory will move in parallel along the axis, and the state parameters will change correspondingly. It can be seen that the selection of $x_{Z}$ has great influence on the terminal dynamic pressure. Thus, the ideal terminal dynamic pressure can be achieved to meet constraints of landing by adjusting $x_{Z}$.

Known that the ideal terminal dynamic pressure is $q_{n}=6891 \mathrm{~N} / \mathrm{m}^{2}$, it can be calculated that $x_{Z}=-2080 \mathrm{~m}$ according to the principle of optimality.

Contrastive Analysis of Simulation Results. In order to verify the realizability of trajectory and the superiority of algorithm, simulation of tracking the nominal trajectory are conducted in six degrees of freedom simulation environment. Figure 4-9 show the comparisons between simulation results and nominal states.

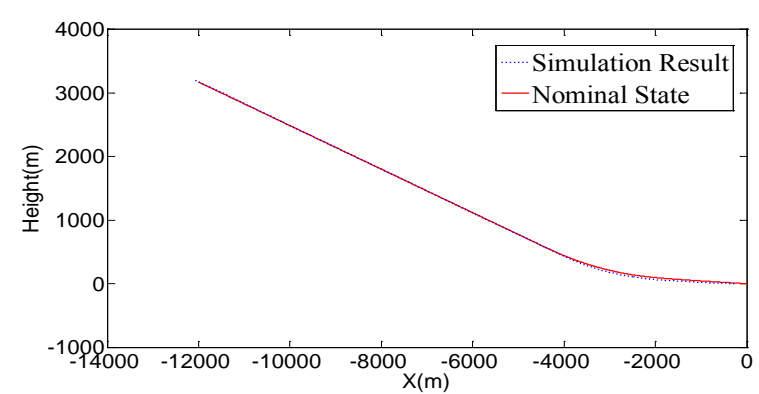

Fig. 4 Comparison of height profiles

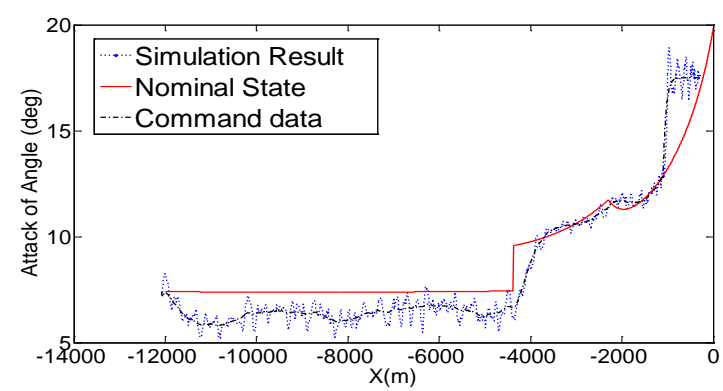

Fig. 6 Comparison of angle of attack profiles

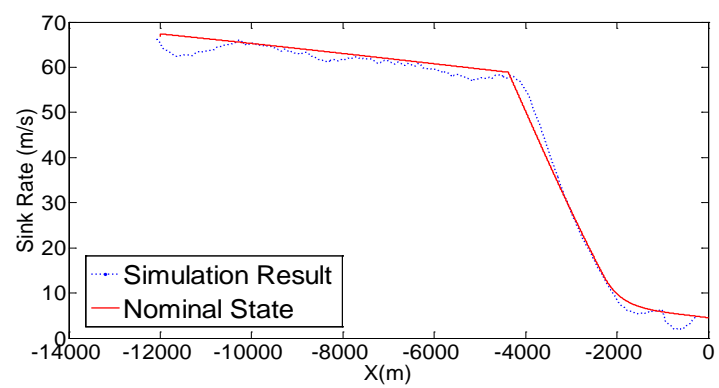

Fig. 8 Comparison of sink rate profiles

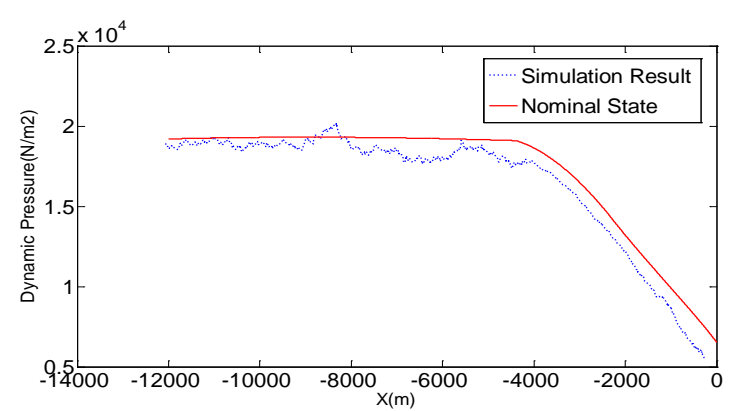

Fig. 5 Comparison of dynamic pressure profiles

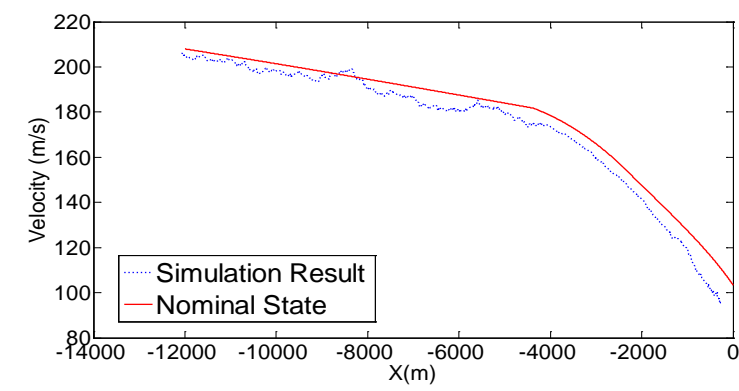

Fig. 7 Comparison of velocity profiles

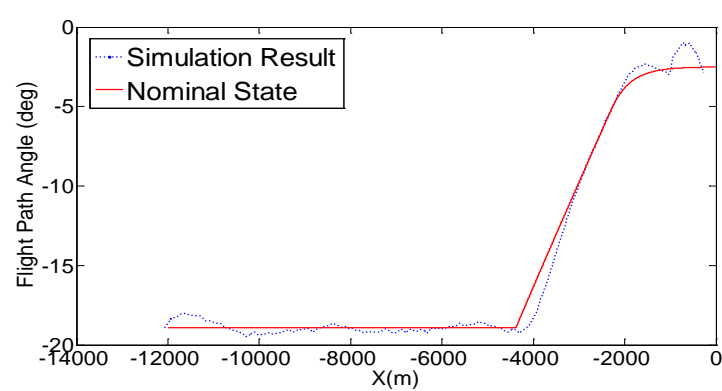

Fig. 9 Comparison of flight path angle profiles From above figures, it can be seen that the stage of steep glide slope takes up a relatively large 
proportion of the whole process of autolanding. In this stage, flight path angle of the vehicle keeps constant, dynamic pressure and angle of attack change slightly, while the air density increases and velocity decreases with the reducing of height. When the height is about $400 \mathrm{~m}$, the vehicle is in phase of circular flare. Its dynamic pressure decreases rapidly while the angle of attack increases rapidly. And there is a jump caused by the abrupt change of acceleration which is inevitable at the junction. At this stage, velocity of the vehicle decreases at a faster rate, and flight path angle reduces gradually. At last, in the stage of shallow glide slope, flight path angle keeps constant until landing on ground.

Above figures suggest that simulation results are essentially in agreement with nominal states, meeting the design requirements and demonstrating the realizability of the trajectory.

There are substantial simplifications in the process of trajectory design, including the vehicle dynamics and aerodynamic parameters. The influence of wind velocity is also not considered. However, final results prove the feasibility and creativeness of the algorithm, as well as the acceptance of the simplification.

\section{Conclusions}

In this paper, a trajectory design methodology is developed for autolanding phase of RLV based on iterative correction algorithm. Compared with the traditional strategy of shuttle, this methodology does not rely on some pre-computed and stored trajectories, and feasible reference profiles of RLV are able to be generated with variations in initial ALI states. The practicability and superiority of the algorithm are proved by simulation results.

However, this methodology of off-line trajectory design has strict constraints on the launch and reentry windows, while onboard trajectory generation can reduce the dependence on predesigned route, broaden restrictions on the initial windows, improve the security and reliability of the system as well as a wider range of uncertainties and better robustness ${ }^{[2,8]}$. Therefore, the technology of online trajectory generation has significant value and is also the further research direction in the filed though it is still in an initial stage at present.

\section{References}

[1] Tsikalas, GM, Space Shuttle Autoland Design, AIAA 82-1604.

[2] Gregg H. Barton, Steven G. Tragesser, Autolanding Trajectory Design for the X-34, AIAA atmospheric flight conference and exhibit, Portland, Oregon, August 9-11, 1999, P15-30.

[3] Sun Chunzhen. Research on Terminal Area Energy Management and Autolanding Technology for Reusable Launch Vehicle[D], Nanjing, NUAA, 2008.

[4] Wu Jianwei, Sun Chunzhen. Survey of Trajectory Design for Reusable Launch Vehicle in the Unpowered Approach and Landing Phase[A]. New Advances in Communications and Information Technology 2011—— the Eighth China Communications Society Symposium[C], Beijing, 2011. 85-88.

[5] Gregg H. Barton. New Methodologies for Assessing the Robustness of the X-34 Autolanding Trajectories[A]. the 24th Annual AAs guidance and control conference[C]. CO: Brckenridge, 2001. 30-41.

[6] Shen Hongliang, Tao Ju, Wei Lixin, Liu Chang. Optimal Design of Autolanding Trajectory for a Space Shuttle[J]. Flight Dynamics, 2004, 22(1). 10-13.

[7] Zhang Jun, Yang Yidong, Cao Dong. Research of Concepts on Autolanding Technology for Space Shuttle[J]. Aerospace Control, 2004, 22(2). 2-5.

[8] Schierman J.D, Hull J R, and Ward D.G, On-line Trajectory Command Reshaping for Reusable Launch Vehicles, AIAA 2003-5439. 